\title{
Coupled-mode theory for photonic band-gap inhibition of spatial instabilities
}

\author{
Damià Gomila and Gian-Luca Oppo \\ Department of Physics, University of Strathclyde, 107 Rottenrow, Glasgow, G4 ONG, United Kingdom
}

(Received 18 February 2005; published 18 July 2005)

\begin{abstract}
We study the inhibition of pattern formation in nonlinear optical systems using intracavity photonic crystals. We consider mean-field models for singly and doubly degenerate optical parametric oscillators. Analytical expressions for the new (higher) modulational thresholds and the size of the "band gap" as a function of the system and photonic crystal parameters are obtained via a coupled-mode theory. Then, by means of a nonlinear analysis, we derive amplitude equations for the unstable modes and find the stationary solutions above threshold. The form of the unstable mode is different in the lower and upper parts of the band gap. In each part there is bistability between two spatially shifted patterns. In large systems stable wall defects between the two solutions are formed and we provide analytical expressions for their shape. The analytical results are favorably compared with results obtained from the full system equations. Inhibition of pattern formation can be used to spatially control signal generation in the transverse plane.
\end{abstract}

DOI: 10.1103/PhysRevE.72.016614 PACS number(s): 42.65.Sf, 89.75.Kd, 05.65.+b, 42.70.Qs

\section{INTRODUCTION}

Photonic crystals (PC's) have been shown to be able to control light in ways that were not possible with conventional optics $[1,2]$. Their remarkable properties stem from the unusual dispersion relation as a result of the periodic modulation of their dielectric properties. Most of the interest in PC's is related to propagation problems. Here the existence of photonic band gaps-i.e., a range of frequencies for which light cannot propagate in the medium-allows for a new way of guiding and localizing light once defects are introduced in the PC's [1,2]. Photonic crystals in combination with nonlinear effects have been also considered for all-optical switching devices [3].

Transverse effects in periodic media have been mainly studied in propagation in planar waveguides with periodic modulation of the refractive index in the transverse direction and arrays of couple waveguides. In the first case attention has been focused on the so-called spatial gap (or Bragg) solitons [4-6], which are intense peaks with frequencies inside the (linear) band gap. This is possible thanks to a shift of the photonic band-gap boundaries due to nonlinear effects: the intense core of a gap soliton can propagate freely in the periodic media, while in its less intense tails, where nonlinearity can be neglected, light is reflected back to the center due to Bragg reflection, sustaining the localized structure. Bragg solitons are usually studied within the coupled-mode theory where only the slowly varying envelope of two counterpropagating beams is considered. Arrays of waveguides are usually studied within the tight-binding approximation, where the system is described by a set of coupled ordinary differential equations describing the dynamics of the guidedmode amplitude in each site. The evanescent coupling between adjacent waveguide gives rise to the so-called discrete diffraction. If nonlinearity is also present, discrete solitons can be formed $[3,4]$.

In this paper we consider a different case, a photonic crystal inside a nonlinear optical cavity-i.e., a system with driving and dissipation. Nonlinear optical cavities typically undergo spatial instabilities leading to the formation of spatial structures similar to what observed in many different fields across science $[7,8]$. In particular, spatial structures in nonlinear optical cavities have important potential applications in photonics such as memories, multiplexing, optical processing, and imaging [9]. Control of spatial structures is then an important issue for the implementation of such devices. In this context, different mechanisms of control have been proposed [10-12]. In particular, Neubecker and Zimmerman reported on experimental pattern formation in presence of an external modulated forcing and observed lockings between the forcing and natural wavelengths [12]. More recently the use of the properties of photonic crystals for the control of optical spatial structures has been considered [13-15]. In [13] we showed how the photonic band gap of a photonic crystal could be used to inhibit a pattern forming instability in a self-focusing Kerr cavity. Pattern formation in an optically injected, photonic-crystal vertical-cavity surfaceemitting laser, electrically biased below threshold, has been observed experimentally in [14]. Finally, in [15], a defect in a photonic crystal has been used to achieve stable emission in broad area lasers.

Here we study the inhibition of pattern formation in a mean-field model for a degenerate optical parametric oscillator with a photonic crystal. We first show that the inhibition mechanism introduced in [13] occurs independently of the type of nonlinearity here being quadratic. Then we introduce an analytical treatment by means of a couple-mode theory that fully explains the phenomenon. A linear stability analysis allows us to determine analytically the new thresholds shifted in parameter space by pattern inhibition and the form of the unstable modes. We show that the band-gap region is divided into two different regions: one in which pattern formation takes place due to an energy concentration at the maxima of the photonic crystal modulation and a different one where the energy concentrates at the minima. We determine amplitude equations for the unstable modes and find the stationary solutions above threshold analytically. We show that this scenario is organized by a codimension- 2 bifurcation point where both modes become simultaneously unstable. We also show that in the band gap, where the pat- 
tern arises with a wave number half the one of the PC, there is bistability between two different "phase-locked" solutions differing by a shift of a PC wavelength in the near field. This bistability stems from the breaking of the translational symmetry by the PC. In large systems this leads to the formation of domains of different "phases" connected by topological walls (or antiphase boundaries) [8]. This is a different kind of (spatial) bistability where the two different "phases" do not differ on the phase of the electric field but on the location of the spatial oscillation in the near field.

\section{MODELS}

A phase-matched double-resonant degenerate optical parametric oscillator (DRDOPO), where both pump and signal fields resonate in the cavity, can be described in the mean-field approximation by [16]

$$
\begin{aligned}
& \partial_{t} B=\Gamma\left[-B+E-A^{2}\right]+i \frac{1}{2} \partial_{x x} B, \\
& \partial_{t} A=-A-i \Delta_{s} A+B A^{*}+i \partial_{x x} A,
\end{aligned}
$$

where $B$ and $A$ are the pump and signal slowly varying amplitudes, $\Gamma$ is the ratio between the pump and signal cavity decay rates, and $E$ is the amplitude of the external pump field. $\Delta_{s}=\Delta+\alpha f(x)$ is the total signal detuning, where $\Delta$ is the average detuning and $f(x)=e^{i k_{p c} x}+e^{-i k_{p c} x}$ describes the spatially dependent contribution of the photonic crystal (Fig. 1).

For the singly resonant DOPO (SRDOPO), where there is no cavity for the pump field, the mean-field equation for the resonating signal is $[17,18]$

$$
\partial_{t} A=-A-i \Delta_{s} A+E A^{*}-|A|^{2} A+i \partial_{x x} A .
$$

\section{LINEAR STABILITY ANALYSIS WITH PERIODIC MEDIA: COUPLE-MODE THEORY}

The linearization around the steady-state homogeneous solution $B^{0}=E$ and $A^{0}=0$ of Eqs. (1) and (2) leads, in both cases, to the same equation for the perturbations of the signal A:

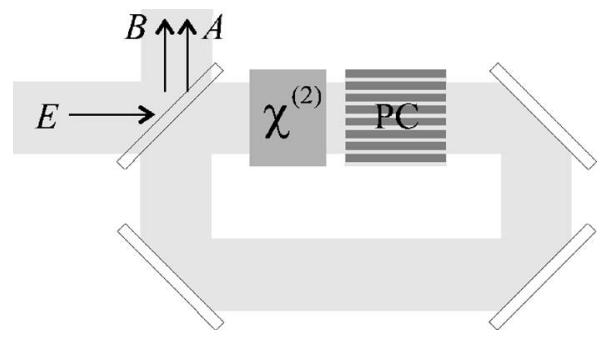

FIG. 1. Scheme of a doubly resonant degenerate optical parametric oscillator with a photonic crystal. It consist of a ring cavity filled with a quadratic $\left(\chi^{(2)}\right)$ medium and a photonic crystal slab (PC). $E$ is the plane-wave input field at frequency $\omega$ partially transmitted into the cavity. The other mirrors are assumed to be perfectly reflecting. $B$ and $A$ are the pump and signal fields at frequencies $\omega$ and $\omega / 2$, respectively. In the singly resonant case only the signal field $A$ resonates with the cavity.

$$
\partial_{t} A=-(1+i \Delta) A-i \alpha f(x) A+E A^{*}+i \partial_{x x} A .
$$

Without the photonic crystal $(\alpha=0)$, the homogeneous solution is stable for $E<1$. Above threshold $(E>1)$ a stripe pattern arises with a wave number $k_{c}=\sqrt{-\Delta}$. Here we restrict ourselves to negative values of the detuning. For positive detuning the system display bistability between homogeneous solutions [18]. For $\alpha \neq 0$ and assuming that the amplitude of the modulation is weak enough, we can write the signal perturbations as a superposition of two waves with opposite transverse wave numbers [6]:

$$
A=A_{+}(x, t) e^{i k_{p c} x / 2}+A_{-}(x, t) e^{-i k_{p c} x / 2},
$$

where $A_{ \pm}(x, y)$ are slow functions of $x$. This is basically equivalent to the so-called coupled-mode theory for propagation of pulses in periodic media [3].

By writing $A_{ \pm}=a_{ \pm}^{+}(t) e^{i k x}+a_{ \pm}^{-}(t) e^{-i k x}$, one obtains a set of coupled linear ordinary differential equations for the amplitudes $a_{ \pm}^{+}\left(a_{ \pm}^{-}\right)$of the Fourier components $\pm k_{p c} / 2+k\left( \pm k_{p c} / 2\right.$ $-k)$ of the perturbations. By defining $\mathbf{F}=\left(a_{+}^{+}, a_{-}^{-*}, a_{-}^{+}, a_{+}^{-*}\right)^{T}$ this set of equations can be written as

$$
\dot{\mathbf{F}}=\mathcal{L} \mathbf{F},
$$

where $\mathcal{L}$ is given by

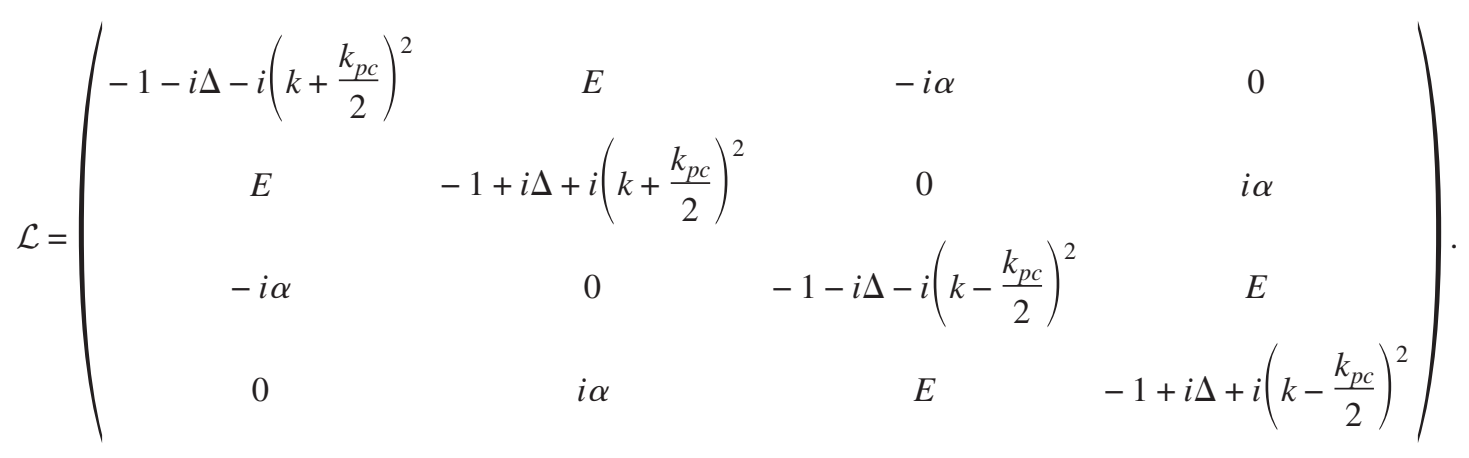




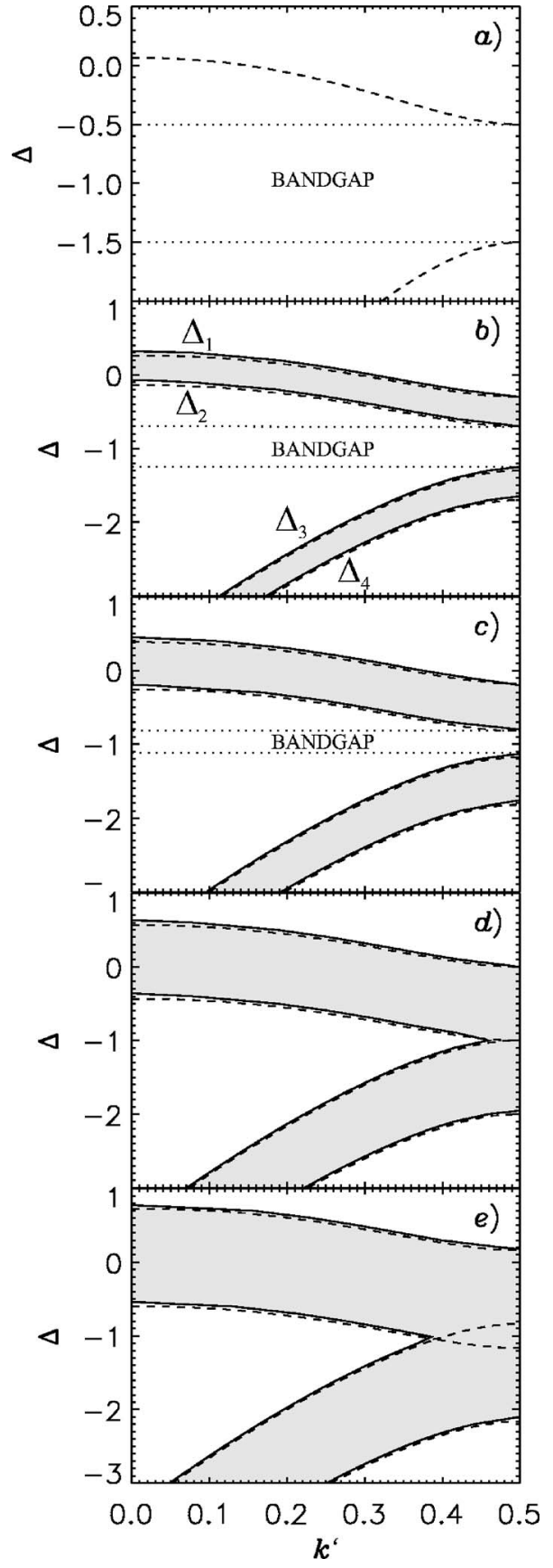

FIG. 2. Marginal stability curves of a DOPO for (a) $E=1.0$, (b) $E=1.02$, (c) $E=1.05$, (d) $E=\sqrt{1+\alpha^{2}}=1.118034$, and (e) $E=1.2$ in the presence of a periodically modulated media $\left(k_{p c}=2, \alpha=0.5\right)$. Solid lines correspond to results obtained with a numerical analysis of the full model equations, while dashed lines are the analytical results from the coupled-mode theory (9). For each value of $\Delta$ the wave numbers within the shaded region are unstable. Note that, for moderate values of the input intensity, there is a gap of detuning values, indicated by the horizontal dotted lines, for which the system is stable.

In this way one reduces the stability analysis of the steady state of the initial partial differential equations with periodic coefficients (1) and (2) to diagonalize the $4 \times 4$ complex matrix $\mathcal{L}$. In the case $k=0$ one has to consider that $a_{ \pm}^{+}=a_{ \pm}^{-}$.

The eigenvalue of $\mathcal{L}$ with largest real part is

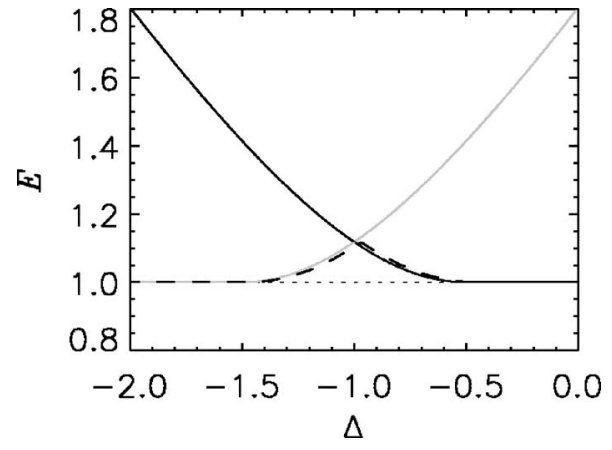

FIG. 3. Pump threshold as a function of the detuning from the theoretical result (10) obtained by means of the coupled- mode theory (solid black and gray lines). The dashed line is the result obtained from a numerical stability analysis of the full model. The dotted line is the threshold for a DOPO without photonic crystal.

$$
\lambda_{1}=-1+\sqrt{E^{2}-\left[k^{2}+\left(\frac{k_{p c}}{2}\right)^{2}+\Delta-\sqrt{\alpha^{2}+4 k^{2}\left(\frac{k_{p c}}{2}\right)^{2}}\right]^{2}}
$$

for $-\left(k_{p c} / 2\right)^{2}<\Delta$ and

$$
\lambda_{2}=-1+\sqrt{E^{2}-\left[k^{2}+\left(\frac{k_{p c}}{2}\right)^{2}+\Delta+\sqrt{\alpha^{2}+4 k^{2}\left(\frac{k_{p c}}{2}\right)^{2}}\right]^{2}}
$$

for $\Delta<-\left(k_{p c} / 2\right)^{2}$. By setting $\lambda_{1}=\lambda_{2}=0$ we obtain four marginal stability curves

$$
\begin{aligned}
& \Delta_{1}(k, E)=-d_{1}(k)+d_{2}(k)+d_{3}(E), \\
& \Delta_{2}(k, E)=-d_{1}(k)+d_{2}(k)-d_{3}(E), \\
& \Delta_{3}(k, E)=-d_{1}(k)-d_{2}(k)+d_{3}(E), \\
& \Delta_{4}(k, E)=-d_{1}(k)-d_{2}(k)-d_{3}(E),
\end{aligned}
$$

where $\quad d_{1}(k)=\left(k_{p c} / 2\right)^{2}+k^{2}, \quad d_{2}(k)=\sqrt{4 k^{2}\left(k_{p c} / 2\right)^{2}+\alpha^{2}}, \quad$ and $d_{3}(E)=\sqrt{E^{2}-1}$.

Figure 2 shows the marginal stability curves for different values of the pump $E$. Note that, due to definition (4), $k$ indicates the relative distance from the wave number of a perturbation to the limit of the first Brillouin zone. Therefore it is convenient to plot $\Delta$ as a function of $k^{\prime}=\left(k_{p c} / 2\right.$ $-k) / k_{p c}$, which is the real wave number of the perturbations in units of $k_{p c}$. Dashed lines are the results from the coupledmode theory (9), while solid lines have been obtained from a numerical stability analysis of the full model-i.e., solving the eigenvalue problem associated with the linear differential operator with periodic coefficients on the right-hand side of Eq. (3) [13]. The coupled-mode theory provides a very good analytical approximation for thresholds and unstable wave numbers, allowing us to predict the existence and size of a band gap in the modulation instability analytically. In the following we analyze the results of the coupled-mode theory in more detail. 
From Eqs. (9) one can evaluate the instability threshold $E_{t h}$ of the fundamental solution as a function of the detuning $\Delta$ (Fig. 3). For $E<1.0$ the steady-state homogeneous solution is stable for all values of the detuning. At $E=1 d_{3}(E)$ $=0$ and the four marginal stability lines given by Eqs. (9) become only two [see Fig. 2(a)], signaling the instability threshold for all values of the detuning outside the band gap $\left[-d_{1}\left(k_{p c} / 2\right)-d_{2}\left(k_{p c} / 2\right)<\Delta<-\left(k_{p c} / 2\right)^{2}-\alpha<-\left(k_{p c} / 2\right)^{2}+\alpha\right.$ $\left.<\Delta<-d_{1}\left(k_{p c} / 2\right)+d_{2}\left(k_{p c} / 2\right)\right]$. In the band gap $-\left(k_{p c} / 2\right)^{2}-\alpha$ $<\Delta<-\left(k_{p c} / 2\right)^{2}+\alpha$, the instability is, however, inhibited and the threshold is higher. The threshold $E=1$ for values of $\Delta$ outside the band gap is slightly underestimated. In the full model, for this value of the pump, the system is still stable [no solid line in Fig. 2(a)]. This is due to the fact that the spatial modulation couples the fundamental wave numbers with their harmonics, which are dumped, introducing an additional source of stability. Harmonics are not taken into account in the couple-mode theory and then the threshold is slightly lower than for the full models (1) and (2).

Increasing further the value of the pump the band gap narrows [Figs. 2(b) and 2(c)], until at $E=\sqrt{1+\alpha^{2}}$ the homogeneous solution becomes eventually unstable for any value of the detuning [Fig. 2(d)]. The threshold value of $E$ as a function of the photonic crystal parameters for any value of $\Delta$ is given by

$$
E_{t h}^{2}(\Delta)=\left\{\begin{array}{cc}
1 & -\left(\frac{k_{p c}}{2}\right)^{2}+\alpha<\Delta \leqslant-d_{1}\left(\frac{k_{p c}}{2}\right)+d_{2}\left(\frac{k_{p c}}{2}\right), \\
1+\left[-\left(\frac{k_{p c}}{2}\right)^{2}+\alpha-\Delta\right]^{2} & -\left(\frac{k_{p c}}{2}\right)^{2}<\Delta \leqslant-\left(\frac{k_{p c}}{2}\right)^{2}+\alpha, \\
1+\left[\left(\frac{k_{p c}}{2}\right)^{2}+\alpha+\Delta\right]^{2} & -\left(\frac{k_{p c}}{2}\right)^{2}-\alpha<\Delta \leqslant-\left(\frac{k_{p c}}{2}\right)^{2}, \\
1 & -d_{1}\left(\frac{k_{p c}}{2}\right)-d_{2}\left(\frac{k_{p c}}{2}\right)<\Delta \leqslant-\left(\frac{k_{p c}}{2}\right)^{2}-\alpha,
\end{array}\right.
$$

and the critical wave number is

$$
k_{c}^{2}(\Delta)=\left\{\begin{array}{cc}
\left(\frac{k_{p c}}{2}\right)^{2}-\Delta-\sqrt{-4\left(\frac{k_{p c}}{2}\right)^{2} \Delta+\alpha^{2}}-\left(\frac{k_{p c}}{2}\right)^{2}+\alpha<\Delta \leqslant-d_{1}\left(\frac{k_{p c}}{2}\right)+d_{2}\left(\frac{k_{p c}}{2}\right), \\
0 & -\left(\frac{k_{p c}}{2}\right)^{2}<\Delta \leqslant-\left(\frac{k_{p c}}{2}\right)^{2}+\alpha, \\
0 & -\left(\frac{k_{p c}}{2}\right)^{2}-\alpha<\Delta \leqslant-\left(\frac{k_{p c}}{2}\right)^{2}, \\
\left(\frac{k_{p c}}{2}\right)^{2}-\Delta-\sqrt{-4\left(\frac{k_{p c}}{2}\right)^{2} \Delta+\alpha^{2}}-d_{1}\left(\frac{k_{p c}}{2}\right)^{2}-d_{2}\left(\frac{k_{p c}}{2}\right)<\Delta \leqslant-\left(\frac{k_{p c}}{2}\right)^{2}-\alpha .
\end{array}\right.
$$

Figure 3 shows the theoretical prediction (10) for the instability threshold of the homogeneous solution (solid lines). The black and gray lines correspond to two different eigenmodes. The first is a mode with energy concentrated in the minima of the photonic crystal, while the second in the maxima (see next section for more details). The dashed line is the threshold for the full system computed as explained above.

The coupled-mode theory accurately capture the features of the inhibition of the modulation instability by the photonic crystal. The fact that the gap of values of the detuning for which pattern formation is inhibited decreases with the intensity of the pump is because nonlinearity always overcomes the linear inhibition by the photonic crystal for suitable high input intensities. This is similar to the phenomenon behind the formation of gap solitons. But in that case the nonlinearity only shifts the position of the band gap, while in our case it narrows it and makes it eventually to disappear.

From Eq. (9) we can also calculate the size $g(E, \alpha)$ of the band gap as a function of the system and photonic crystal parameters:

$$
g(E, \alpha)=\Delta_{2}(k=0, E)-\Delta_{3}(k=0, E)=2\left(\alpha-\sqrt{E^{2}-1}\right) .
$$

The size of the band gap is proportional to the amplitude of the modulation $\alpha$ and becomes smaller by increasing the pump $E$ (Fig. 2), eventually disappearing for $E=\sqrt{1+\alpha^{2}}$ [Fig. 2(d)]. For $E>1+\alpha^{2}$ the homogeneous solution is unstable for any value of $\Delta$ [Fig. 2(e)]. Figure 4 shows how the width of the band gap (white region) changes as a function of the amplitude of the modulation $\alpha$ for a fixed value of the pump $E$. The presence of the modulated medium opens an entirely 


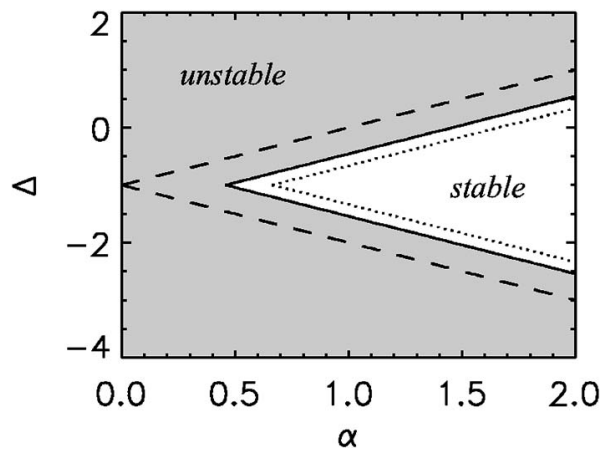

FIG. 4. Stability diagram of the steady-state homogeneous solution for $E=1.1$ (solid line). The system is unstable in the shadowed region. The dashed (dotted) line shows the boundary of the stable region for $E=1.0(E=1.2)$.

new stable region. Figure 4 should be compared with Fig. 6 of [13] showing that pattern inhibition is independent of the form of the nonlinearity.

Since for negative signal detunings, in the absence of PC, down-conversion takes place at a finite wave number, the inhibition mechanism can be used to spatially control the generation of signal in a way analogous to that explained in [13]. If we set our system in a parameter region where pattern formation is inhibited, the inclusion of a defect in the PC will lead to a spot of signal generation as shown in Fig. 5.

\section{WEAKLY NONLINEAR ANALYSIS: AMPLITUDE EQUATIONS FOR PATTERN FORMATION IN PERIODIC MEDIA}

In this section we study by means of a multiple scales analysis the solutions that appear above threshold for values of the detuning inside the band gap. Above threshold nonlinear terms have to be considered since they saturate the linear growth induced by the instability. In the following, for the sake of simplicity, we consider the SRDOPO only. The qualitative results also apply to the case of the DOPO. By including the nonlinear terms from Eqs. (2) in (5) we obtain

$$
\dot{\mathbf{F}}=\mathcal{L} \mathbf{F}-\mathcal{W}(\mathbf{F}),
$$

where $\quad \mathcal{W}=\left[\left(a_{+}^{+} a_{+}^{-*}+2 a_{-}^{-*} a_{-}^{+}\right) a_{+}^{+},\left(2 a_{-}^{+} a_{-}^{-*}+a_{+}^{-*} a_{+}^{+}\right) a_{-}^{-*}\left(a_{-}^{+} a_{-}^{-*}\right.\right.$ $\left.\left.+2 a_{+}^{-*} a_{+}^{+}\right) a_{-}^{+},\left(2 a_{+}^{+} a_{+}^{-*}+a_{-}^{-*} a_{-}^{+}\right) a_{+}^{-*}\right]^{T}$ is a nonlinear function of F.

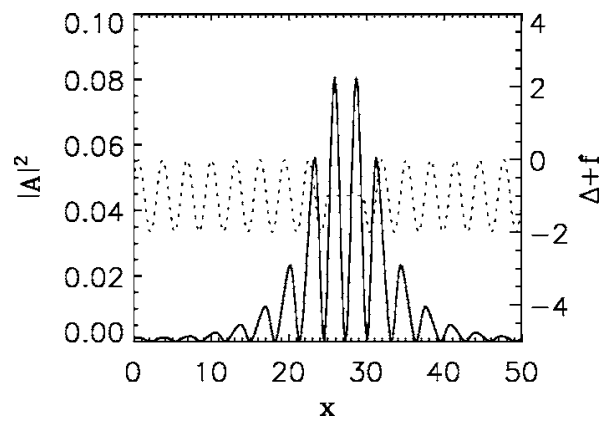

FIG. 5. Localized spot of signal generation due to a defect in the photonic crystal. Here $E=1.08, \Delta=-1, k_{p c}=2$, and $\alpha=0.5$.
In the band gap, the critical wave number is always $k_{c}$ $=0$ independently of the detuning. We recall that in this case one has to consider $a_{ \pm}^{+}=a_{ \pm}^{-}$. In the following we will discuss the lower $\left[-\left(k_{p c} / 2\right)^{2}-\alpha \leqslant \Delta \leqslant-\left(k_{p c} / 2\right)^{2}\right]$ and upper $\left[-\left(k_{p c} / 2\right)^{2} \leqslant \Delta \leqslant-\left(k_{p c} / 2\right)^{2}+\alpha\right]$ halves of the band gap separately.

\section{A. Lower-half part}

Assuming the following scaling [16]:

$$
\begin{gathered}
\mathbf{F}=\epsilon \mathbf{F}_{1}+\epsilon^{3} \mathbf{F}_{3}, \\
E=E_{t h}+\epsilon^{2} E_{2}, \\
T=\epsilon^{2} t, \\
X=\epsilon^{2} x,
\end{gathered}
$$

and substituting Eq. (14) into Eq. (13), at order $\epsilon$ one obtains $\mathbf{F}_{1}=F_{1} \mathbf{v}_{1}$, where $\mathbf{v}_{1}$ is the critical eigenmode of $\mathcal{L}$ associated with $\lambda_{2}\left(\mathcal{L} \mathbf{v}_{1}=0\right.$ and $\left.\left|\mathbf{v}_{1}\right|=1\right)$ and $F_{1}$ is its real amplitude. In the near field, $\mathbf{v}_{1}$ has the form $\left[\left(1+E_{t h}\right.\right.$ $\left.\left.-i \sqrt{E_{t h}^{2}-1}\right) / \sqrt{2 E_{t h}\left(1+E_{t h}\right)}\right] \cos \left(k_{p c} x / 2\right)$.

The solvability condition at order $\epsilon^{3}$ yields to the following equation for the real amplitude $\tilde{A}_{1}=\epsilon F_{1}$ of the unstable mode:

$$
\partial_{t} \widetilde{A}_{1}=\sqrt{E_{t h}^{2}-1} \partial_{x}^{2} \widetilde{A}_{1}+E_{t h}\left(E-E_{t h}\right) \tilde{A}_{1}-\frac{3}{4} \widetilde{A}_{1}^{3} .
$$

The amplitude equation (15) is equivalent to the one obtained from a secondary instability at twice the spatial period of a cellular pattern [19], except for the fact that in our case the translational invariance has been broken by the presence of the photonic crystal. The homogeneous steady-state solution of Eq. (15) is $\widetilde{A}_{1}= \pm 2 \sqrt{E_{t h}\left(E-E_{t h}\right) / 3}$. The solution of Eq. (2) is then

$$
A(x)= \pm \sqrt{\frac{2}{3}} \sqrt{\frac{E-E_{t h}}{1+E_{t h}}}\left(1+E_{t h}-i \sqrt{E_{t h}^{2}-1}\right) \cos \left(\frac{k_{p c}}{2} x\right) .
$$

The bifurcation diagram and spatial form of this solution are shown in Fig. 6. The plus and minus solutions (16) are created in a pitchfork bifurcation. The analytical solution (16) is in very good agreement with the stationary solution of the full model computed numerically. Note that, despite being completely equivalent, the plus and minus solutions of Eq. (16) are not the same. One corresponds to the other shifted by a wavelength of the photonic crystal. In a system with translational invariance the position of a solution that breaks such symmetry is undetermined. The photonic crystal periodicity selects just two of this continuum of possible solutions. These two "frequency-locked" solutions differ now by a shift of a photonic crystal wavelength in the transverse position. This situation is the spatial analog of an oscillatory system forced at twice its natural frequency [20]. In a large system and starting from arbitrary initial conditions, 

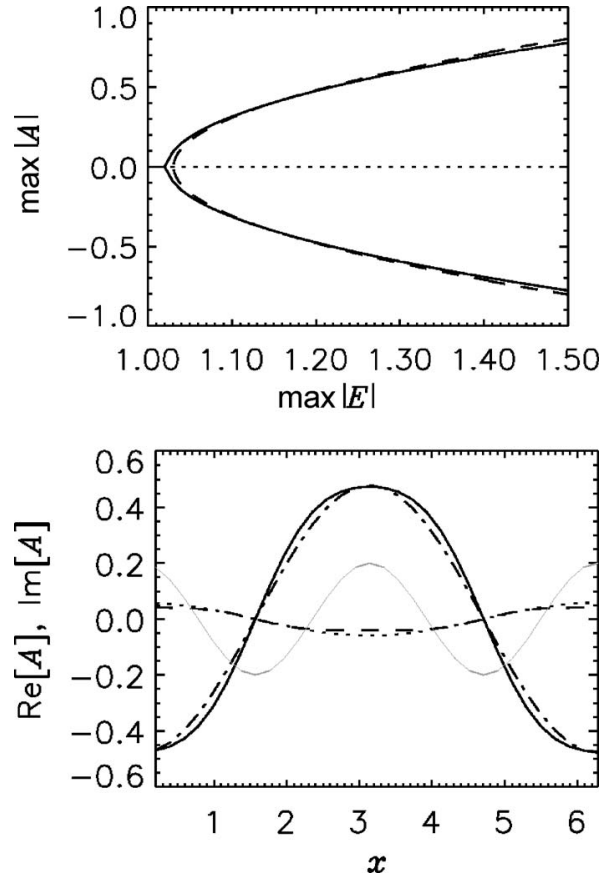

FIG. 6. Left: bifurcation diagram for the pattern arising above threshold in the lower-half of the band gap. Here $\Delta=-1.2$. For this value of the detuning $E_{t h}=1.04403$. Right: real (solid line) and imaginary (dashed line) parts of the pattern solution of the full model for $E=1.2$. The dot-dashed and dotted lines correspond to the real and imaginary parts of the pattern from the coupled-mode theory. The gray solid line illustrates the modulation of the photonic crystal for comparison.

some sections of the system will attain the first solution while others will move to the second one, leading to the formation of domain walls. This is illustrated in Fig. 7. The solid line is the result of a simulation of the full model (2) starting from a random initial condition. The dashed line is the steady state solution of Eq. (15) connecting the two (plus and minus) homogeneous solutions [8] times the critical eigenmode $\mathbf{v}_{1}$ in the near field:

$$
\begin{aligned}
A_{1}(x)= & \sqrt{\frac{2}{3}} \sqrt{\frac{E-E_{t h}}{1+E_{t h}}}\left(1+E_{t h}-i \sqrt{E_{t h}^{2}-1}\right) \\
& \times \tanh \left[\sqrt{\frac{E_{t h}\left(E-E_{t h}\right)}{2}} \frac{x}{\left(E_{t h}^{2}-1\right)^{1 / 4}}\right] \cos \left(\frac{k_{p c}}{2} x\right) .
\end{aligned}
$$

The dot-dashed line in Fig. 7 is the envelope of Eq. (17). The analytical result (17) is in very good agreement with the domain wall obtained from the numerical simulations of the full model (2).

\section{B. Upper-half part}

In the upper-half part of the photonic band gap the critical mode $\mathbf{v}_{1}$ is associated with $\lambda_{1}$ and has the form $\left\{\left[\sqrt{E_{t h}^{2}-1}\right.\right.$ $\left.\left.+i\left(E_{t h}-1\right)\right] / \sqrt{2 E_{t h}\left(E_{t h}-1\right)}\right\} \sin \left(k_{p c} x / 2\right)$. As in the previous case, we obtain a similar amplitude equation for the real amplitude $\widetilde{A}_{1}$ of the unstable mode:
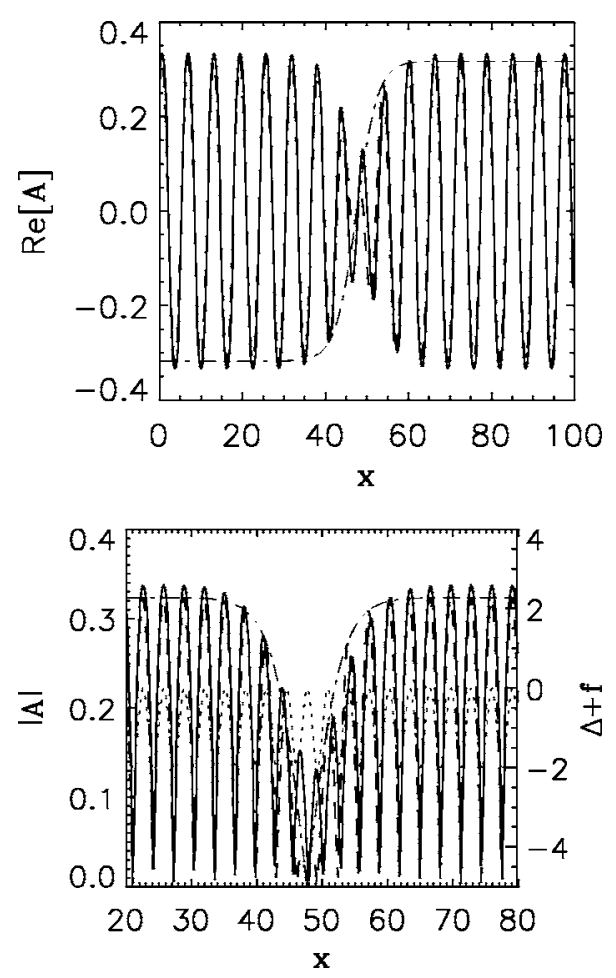

FIG. 7. Two domains corresponding to the plus and minus sign solutions (16) separated by domain walls. The final state is the result of a numerical simulation starting from an arbitrary initial condition. Left: real part of the field. Right: close-up of the intensity around a domain wall. The dotted line shows the modulation of the photonic crystal. Here $\Delta=-1.1, E=1.15, \alpha=0.5$, and $k_{p c}=2.0$.

$$
\partial_{t} \tilde{A}_{1}=\sqrt{E_{t h}^{2}-1} \partial_{x}^{2} \widetilde{A}_{1}+\left(E-E_{t h}\right) \tilde{A}_{1}-\frac{3}{4} \widetilde{A}_{1}^{3} .
$$

In this case the pattern solutions is

$$
\begin{aligned}
A_{1}(x)= & \pm \sqrt{\frac{2}{3}} \sqrt{\frac{E-E_{t h}}{E_{t h}\left(E_{t h}-1\right)}}\left[\sqrt{E_{t h}^{2}-1}\right. \\
& \left.+i\left(E_{t h}-1\right)\right] \sin \left(\frac{k_{p c}}{2} x\right) .
\end{aligned}
$$

The bifurcation diagram and spatial form of this solution is shown in Fig. 8. The analytical solution (19) is in very good agreement with the stationary solution of the full model computed numerically. As in the previous case, in large systems, fronts between the plus and minus solutions are formed. The shape of the front is given in this case by

$$
\begin{aligned}
A_{1}(x)= & 2 \sqrt{\frac{2}{3}} \sqrt{\frac{E-E_{t h}}{E_{t h}\left(E_{t h}-1\right)}}\left[\sqrt{E_{t h}^{2}-1}\right. \\
& \left.+i\left(E_{t h}-1\right)\right] \tanh \left[\sqrt{\frac{E-E_{t h}}{2}} \frac{x}{\left(E_{t h}^{2}-1\right)^{1 / 4}}\right] \\
& \times \sin \left(\frac{k_{p c}}{2} x\right) .
\end{aligned}
$$

Note that while the cosine solution in the lower-half part of the band gap has the maxima of the intensity at the 

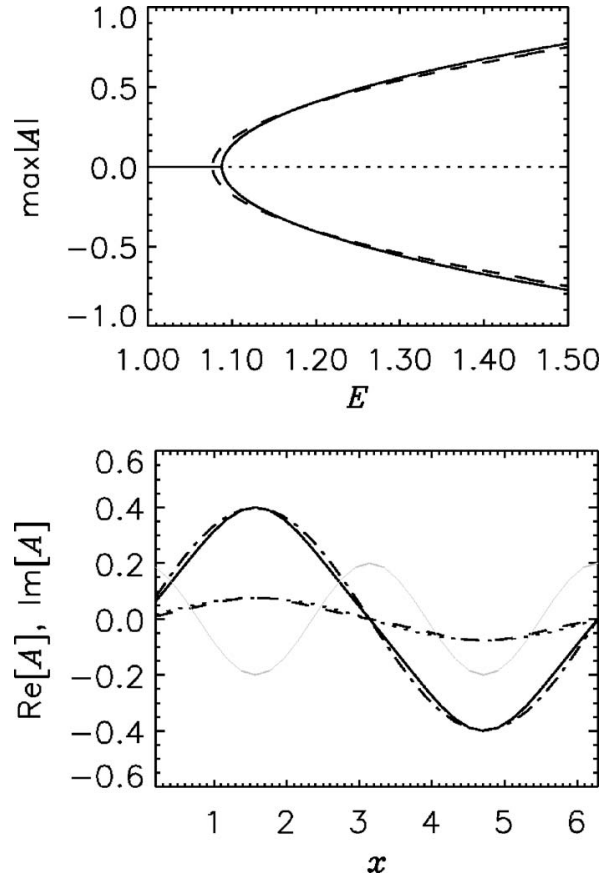

FIG. 8. Left: bifurcation diagram for the pattern arising above threshold in the lower half of the band gap. Here $\Delta=-0.9$. For this value of the detuning $E_{t h}=1.07703$. Right: real (solid line) and imaginary (dashed line) parts of the pattern solution of the full model for $E=1.2$. The dot-dashed and dotted lines correspond to the real and imaginary parts of the pattern from the coupled-mode theory. The gray solid line illustrates the modulation of the photonic crystal for comparison.

maxima of the photonic crystal modulation, the sine solution in the upper part has them at the minima. This different distribution of energy in the photonic crystal is at the basis of the creation of the band gap. An interesting point is the middle of the band gap $\left[\Delta=-\left(k_{p c} / 2\right)^{2}\right]$. At this particular value of the detuning, for $E=E_{t h}=\sqrt{1+\alpha^{2}}$ both $\lambda_{1}$ and $\lambda_{2}$ vanish, and the sine and cosine modes become simultaneously unstable. This is a codimension-2 point where Eqs. (15) and (18) become coupled. The unfolding of such a critical point is, however, beyond the scope of this paper and it is left for future investigation.

\section{CONCLUSIONS}

In this paper we have studied pattern formation in nonlinear optical cavities in presence of a photonic crystal-i.e., a spatial modulation of the refractive index. The linear phenomenon of the band gap inhibits pattern formation for a certain range of cavity detunings (band gap). For high enough intensities nonlinearity finally overcomes the inhibition by the photonic crystal and a pattern arises. By means of a couple-mode theory approach we have obtained analytical expressions for the new (shifted) threshold and the form of the unstable modes. The band gap is naturally divided in two halves, the lower-half one in which the unstable mode has a cosine shape-i.e., its intensity maxima are in correspondence with the maxima of the photonic crystal modulationand the upper-half where the unstable mode is a sine; the intensity maxima are in correspondence with the minima of the photonic crystal modulation. By means of a multiplescale analysis we also found the pattern solution above threshold. In each part of the band gap there is bistability between the plus and minus cosine or sine solutions. This bistability stems from the breaking of the translational symmetry of the photonic crystal. In large systems domain wall between this two solutions are typically form. The shape of the defect wall is given by an hyperbolic tangent. While the particular form of the coefficients are model dependent, the shape of the unstable modes and the splitting of the band gap in two different regions are generic. We have checked that the same phenomenon is present in a completely different model: namely, the Kerr cavity model studied in [13]. Finally we also have shown that photonic crystal can be useful to engineer particular spatial signal outputs in frequency down conversion. An interesting extension of this work is to consider the case with two transverse dimensions. In this case, some of the features studied here, such us the two different modes in each part of the band gap, will remain the same, while new features, like the coupling of the geometry of the photonic crystal and that of the spontaneous pattern, will come into play.

\section{ACKNOWLEDGMENTS}

We thank A. J. Scroggie for useful discussions. We acknowledge financial support from EPSRC (Grant Nos. GR S28600/1 and GR R04096/01), SGI, the Royal Society, Leverhulme Trust, and the European Commission (FunFACS).
[1] J. D. Joannopoulos, R. D. Meade, and J. N. Winn, Photonic Crystals (Princeton University Press, Singapore, 1995); J. D. Joannopoulos, P. R. Villeneuve, and S. Fran, Nature (London) 386, 143 (1997).

[2] J. C. Knight, Nature (London) 424, 846 (2003).

[3] Nonlinear Photonic Crystals, edited by R. E. Slusher and B. J. Eggleton (Springer, Berlin, 2003).

[4] Y. S. Kivshar and G. P. Agrawal, Optical Solitons: From Fi- bers to Photonic Crystals (Academic Press, San Diego, 2003); Spatial Solitons, edited by S. Trillo and W. Torruellas (Springer, Berlin, 2001).

[5] R. F. Nabiev, P. Yeh, and D. Botez, Opt. Lett. 18, 1612 (1993).

[6] A. V. Yulin, D. V. Skryabin, and W. J. Firth, Phys. Rev. E 66, 046603 (2002).

[7] M. C. Cross and P. C. Hohenberg, Rev. Mod. Phys. 65, 851 (1993). 
[8] D. Walgraef, Spatio-temporal Pattern Formation (SpringerVerlag, New York, 1997).

[9] W. J. Firth and C. O. Weiss, Opt. Photonics News 13(2), 54 (2002); S. Barland et al., Nature (London) 419, 699 (2002).

[10] R. Martin, A.J. Scroggie, G.-L. Oppo, and W.J. Firth, Phys. Rev. Lett. 77, 4007 (1996).

[11] G. Harkness et al., Opt. Photonics News 9, 44 (1998).

[12] R. Neubecker and A. Zimmermann, Phys. Rev. E 65, 035205(R) (2002).

[13] D. Gomila, R. Zambrini, and G.-L. Oppo, Phys. Rev. Lett. 92, 253901 (2004).

[14] M. Dabbicco, T. Maggiopinto, and M. Brambilla, Appl. Phys. Lett. 86, 021116 (2005).
[15] T. S. Kim, A. J. Danner, D. M. Grasso, E. W. Young, and K. D. Choquette, Electron. Lett. 40, 1340 (2004).

[16] G.-L. Oppo, M. Brambilla, and L. A. Lugiato, Phys. Rev. A 49, 2028 (1994); G.-L. Oppo et al., J. Mod. Opt. 41, 1151 (1994).

[17] S. Longhi, J. Mod. Opt. 43, 1089 (1996).

[18] G.-L. Oppo, A. J. Scroggie, and W. J. Firth, Phys. Rev. E 63, 066209 (2001).

[19] P. Coullet and G. Iooss, Phys. Rev. Lett. 64, 866 (1990).

[20] P. Coullet, J. Legga, B. Houchmanzadeh, and J. Lajzerowicz, Phys. Rev. Lett. 65, 1352 (1990); P. Coullet and K. Emilsson, Physica D 61, 119 (1992). 